Journal of

Business \&

Economic

Statistics

는

\section{Journal of Business \& Economic Statistics}

ISSN: 0735-0015 (Print) 1537-2707 (Online) Journal homepage: https://www.tandfonline.com/loi/ubes20

\title{
Causal Pitfalls in the Decomposition of Wage Gaps
}

\section{Martin Huber}

To cite this article: Martin Huber (2015) Causal Pitfalls in the Decomposition of Wage Gaps, Journal of Business \& Economic Statistics, 33:2, 179-191, DOI: 10.1080/07350015.2014.937437

To link to this article: https://doi.org/10.1080/07350015.2014.937437

Accepted author version posted online: 10

Jul 2014.

Published online: 10 Jul 2014.

Submit your article to this journal $₫$

Џ Article views: 719

View Crossmark data

47 Citing articles: 5 View citing articles 진 


\title{
Causal Pitfalls in the Decomposition of Wage Gaps
}

\author{
Martin Huber \\ University of Fribourg, 1700 Fribourg, Switzerland (martin.huber@unifr.ch)
}

\begin{abstract}
The decomposition of gender or ethnic wage gaps into explained and unexplained components (often with the aim to assess labor market discrimination) has been a major research agenda in empirical labor economics. This article demonstrates that conventional decompositions, no matter whether linear or nonparametric, are equivalent to assuming a (probably too) simple model of mediation (aimed at assessing causal mechanisms) and may therefore lack causal interpretability. The reason is that decompositions typically control for post-birth variables that lie on the causal pathway from gender/ethnicity (which are determined at or even before birth) to wage but neglect potential endogeneity that may arise from this approach. Based on the newer literature on mediation analysis, we therefore provide more attractive identifying assumptions and discuss nonparametric identification based on reweighting.
\end{abstract}

KEY WORDS: Causal mechanisms; Mediation; Wage decomposition.

\section{INTRODUCTION}

The decomposition of empirically observed wage gaps across gender or ethnicity has continued to attract substantial attention in labor economics. The idea is to disentangle the total gap into an explained component that can be attributed to differences in (observed) labor market relevant characteristics such as education and occupation and an unexplained remainder, which is often interpreted as discrimination. In addition to the classical linear decomposition of Blinder (1973) and Oaxaca (1973), the use of more flexible nonparametric decomposition methods has been proposed, for instance by DiNardo, Fortin, and Lemieux (1996), Barsky et al. (2002), Frölich (2007), Mora (2008), and Nopo (2008). Furthermore, the literature has also moved from the mere assessment of mean gaps to decompositions at particular quantiles in the outcome distribution, see Juhn, Murphy, and Pierce (1993), DiNardo, Fortin, and Lemieux (1996), Machado and Mata (2005), Melly (2005), Firpo, Fortin, and Lemieux (2007, 2009), and Chernozhukov, Fernandez-Val, and Melly (2009).

These advancements in the estimation of decompositions stand in stark contrast to the lack of a formal identification theory in many if not most studies, as also pointed out by Fortin, Lemieux, and Firpo (2011): "In econometrics, the standard approach is to first discuss identification(...)and then introduce estimation procedures to recover the object we want to identify. In the decomposition literature, most papers jump directly to the estimation issues (i.e., discuss procedures) without first addressing the identification problem."

To close this gap, the main contribution of this article is to shed light on the (plausibility of the) identifying assumptions required for disentangling explained and unexplained components. It will be demonstrated that conventional decompositions of gender and ethnic wage gaps can be equivalently expressed as a system of equations that corresponds to a (probably too) simple model for mediation analysis, which allows explicating the rather strong identifying assumptions underlying most of the literature. Mediation analysis, as outlined in the seminal article of Baron and Kenny (1986), aims at disentangling the causal mechanisms through which an explanatory variable affects an outcome of interest, with mediators being intermediate outcomes lying on the causal pathway between the explanatory variable and the outcome. Applied to the context of wage decompositions, gender and ethnicity can be regarded as variables that stand at the beginning of any individual's causal chain affecting wage, because they are determined at or prior to birth. Education, occupation, work experience, etc., are all mediators because they occur later in life and are thus potentially driven by gender and ethnicity, while the mediators themselves likely affect wage. If we accept this causal structure, which in terms of the time line of events in life appears to be the only reasonable choice, then the explained component of the wage gap can be shown to correspond to the "indirect" wage effect of gender or ethnicity that operates through these mediators. Conversely, the unexplained component equals the "direct" effect of gender or ethnicity on wage that either is inherently direct or operates through unobserved mediators (e.g., discrimination).

Disentangling direct and indirect effects requires conditioning on the mediators while also controlling for confounders jointly related with the outcome, the mediators, and/or the initial explanatory variable, see Judd and Kenny (1981) for an early discussion of the confounding problem in mediation. The identification issue arising in many decompositions is that they merely incorporate mediators but typically neglect potential confounders, which may jeopardize the causal interpretability of the explained and unexplained components. For example, assume that even conditional on the initial variable gender or ethnicity, family background (such as parents' education) affects both the mediator education and the outcome variable wage, for instance through unobserved personality traits like self-esteem

(c) 2015 American Statistical Association Journal of Business \& Economic Statistics

April 2015, Vol. 33, No. 2

DOI: 10.1080/07350015.2014.937437

Color versions of one or more of the figures in the article can be found online at www.tandfonline.com/r/jbes. 
(see Heckman, Stixrud, and Urzua 2006). ${ }^{1}$ Then, using education in the decomposition without controlling for family background generally biases the explained and unexplained components in the wage decomposition. The reason is that education is itself already an intermediate outcome such that conditioning on it without accounting for confounders is likely to introduce bias, a problem thoroughly discussed in Rosenbaum (1984) and Robins and Greenland (1992), among many others.

It is important to note that theoretical cases in which conventional decompositions bear a causal interpretation do exist, but may appear unrealistic. The first scenario is that there are no confounders of gender/ethnicity-henceforth referred to as group variables - and/or the mediators, which seems quite restrictive. The second scenario is a reversal of the causal chain of the group variable and the mediators. If the observed characteristics related to the explained component were determined prior to the group variable (rather than vice versa) and included all confounders of the group variable, the decomposition would satisfy the kind of conditional independence assumption frequently imposed in the treatment evaluation literature, see Rosenbaum and Rubin (1983) and Imbens (2004). Fortin, Lemieux, and Firpo (2011) pointed out that in this case, the unexplained component corresponds to the "treatment effect on the treated" or "nontreated" (depending on the reference group) of the treatment evaluation literature, while the explained component reflects the selection bias into the group variable. Unfortunately, this framework appears ill-suited for decompositions related to gender and ethnicity, which naturally stand at the beginning of any causal chain in an individual's life. Even potentially interesting group variables occurring later in life like unionization may at least partially affect variables we would consider important for the explained component, such as tenure with the current employer.

Therefore, as a second contribution this article suggests the use of arguably more realistic identifying assumptions for wage decompositions (by borrowing from the newer literature on mediation analysis) that allow for confounding of the group variable and mediators and also discusses nonparametric identification. Given the satisfaction of the assumptions, the unexplained and explained components bear a clear causal interpretation. Identification relies on a sequential ignorability assumption as (among others) discussed in Imai, Keele, and Yamamoto (2010), implying that all confounders of the group variable and the mediators are observed. Using the approach suggested in Huber (2013), the explained and unexplained components are then nonparametrically identified by reweighting observations as a function of their propensities to be in a group (i) given the confounders and (ii) given the confounders and mediators. We provide a brief simulation study that conveys the identification issues in wage decompositions. As an empirical illustration, we use the National Longitudinal Survey of Youth 1979 (NLSY79) to estimate the decomposition of the ethnic wage gap among males in the year 2000. Our results are not robust to the choice of identifying assumptions, suggesting that conventional decompositions may be substantially biased. Albeit more general than the standard

\footnotetext{
${ }^{1}$ Analyzing Brazilian earnings data, Lam and Schoeni (1993) argued in a similar manner that family background is correlated with unobserved worker characteristics that affect earnings, while also driving education.
}

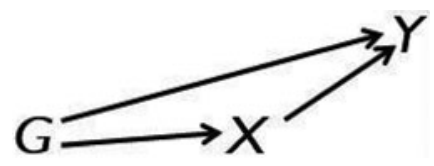

Figure 1. A graphical representation of the decomposition.

decomposition, it has to be stressed that the assumptions proposed in this article are not innocuous either. Besides requiring the observability of all confounders, they also rule out that some confounders of the mediators are themselves a function of the group variable. Alternative identification strategies allowing for the latter case are therefore also briefly discussed and may be considered in future research on wage decompositions.

The remainder of this article is organized as follows. Section 2 shows the equivalence of conventional decompositions and simple mediation models, given that the group variable precedes the observed characteristics (mediators). Second, it discusses arguably more realistic identifying assumptions and identification based on reweighting. Section 3 provides a brief simulation study, while Section 4 presents an application to the NLSY79. Section 5 concludes.

\section{MODELS AND IDENTIFYING ASSUMPTIONS}

\subsection{Notation and Definition of Parameters}

For reasons discussed in Section 1, we will assume throughout that the group variable precedes the observed characteristics we would like to adjust for in the decomposition. Let $G$ denote the binary group variable (e.g., female/male or black/white), $Y$ the outcome of interest (e.g., log wage) and $X$ the vector of observed characteristics (e.g., age, education, work experience, occupation, industry). $X_{k}$ corresponds to the $k$ th element in $X$ with $k \in\{1,2, \ldots, K\}$ and $K$ being the number of characteristics so that $X=\left[X_{1}, \ldots, X_{K}\right]$. A graphical representation of the causal mechanism considered is given in Figure 1, where the arrows represent a causal relation. $G$ (e.g., gender) has an effect on the mediators $X$ (e.g., education and occupation) which themselves affect $Y$ (log wage). At the same time, $G$ also influences $Y$ "directly." However, it is important to note that this causal path need not be inherently direct, but may also or even exclusively include (unobserved) mediators not appearing in $X$. For example, gender and ethnicity most likely affect the perception of individual traits by decision makers in the labor market (see the discussion in Greiner and Rubin 2011), which in turn may entail discriminatory behavior that adds to the unexplained component in wage gaps. ${ }^{2}$

To formally define the causal parameters of interest, we denote by $Y(g)$ and $X(g)$ the potential outcome and mediators

${ }^{2}$ Even if we followed the principle "No causation without manipulation" and were uncomfortable with investigating causal effects of immutable characteristics like gender and ethnicity, see Greiner and Rubin (2011), acknowledging that the causal relations in Figure 1 run through perceived treats may render wage decompositions nevertheless interesting. The reason is that in contrast to factual gender and ethnicity, the perception of these (or related) characteristics may in principal be (experimentally) manipulable, see, for instance, Bertrand and Mullainathan (2004). 
when exogenously setting $G$ to $g$, with $g \in\{1,0\}$ (see, for instance, Rubin 1974 for an introduction to the potential outcome framework). $E(Y(1))-E(Y(0))$ therefore gives the total average causal effect of $G$ on $Y$, represented by the sum of direct and indirect (i.e., operating through $X$ ) effects in Figure 1. $E(X(1))-E(X(0))$, on the other hand, is the average causal effect of $G$ on $X$ (represented by the arrow of $G$ to $X$ in Figure 1), so to speak the "first stage" of the indirect effect. Furthermore, assume that the conditional mean of the potential outcome $Y(g)$ given $X$ is characterized by the following linear model:

$$
E(Y(g) \mid X)=c(g)+\sum_{k=1}^{K} X_{k} \beta_{k}(g), \quad g \in\{1,0\} .
$$

$c(g)$ is a constant and the vector $\beta(g)=\left[\beta_{1}(g), \ldots, \beta_{K}(g)\right]$ contains the (linear) effects of the mediators on the potential outcome, which (as implied by our notation) may differ across $g=1,0$ and therefore allow for group-mediator-interaction effects. The sum of elements in $\beta(g)$ corresponds to the "second stage" of the indirect effect, namely the causal arrow from $X$ to $Y$ in Figure 1, which is permitted to depend on the group state. After having defined all ingredients of our causal framework, the total causal effect can be decomposed into the indirect effect explained by the mediators, denoted by $\psi$, and the unexplained direct effect, denoted by $\eta$, as follows:

$$
\begin{aligned}
E(Y(1))-E(Y(0)) & \\
= & \underbrace{\sum_{k=1}^{K}\left[E\left(X_{k}(1)\right)-E\left(X_{k}(0)\right)\right] \beta_{k}(1)}_{\text {explained component }(\psi)} \\
& +\underbrace{(c(1)-c(0))+\sum_{k=1}^{K} E\left(X_{k}(0)\right)\left(\beta_{k}(1)-\beta_{k}(0)\right)}_{\text {unexplained component }(\eta)} .
\end{aligned}
$$

To see this, first note that $E(Y(1))-E(Y(0))=E[Y(1) \mid$ $E(X(1))]-E[Y(0) \mid E(X(0))]$ under the linear model postulated in (1). Second, $E[Y(1) \mid E(X(1))]-E[Y(0) \mid E(X(0))]=$ $\{E[Y(1) \mid E(X(1))]-E[Y(1) \mid E(X(0))]\}+\{E[Y(1) \mid E(X(0))]$ $-E[Y(0) \mid E(X(0))]\}$ by subtracting and adding $E[Y(1) \mid$ $E(X(0))]$. Third, again by equation (1), $E[Y(1) \mid E(X(1))]-$ $E[Y(1) \mid E(X(0))]=\psi \quad$ and $\quad E[Y(1) \mid E(X(0))]-E[Y(0) \mid$ $E(X(0))]=\eta$.

\subsection{Linking Conventional Wage Gap Decompositions to Mediation Models}

We demonstrate that the linear decomposition of Blinder (1973) and Oaxaca (1973) (i) can be equivalently expressed as a rather simple mediation model and (ii) fails to identify the causal paths in Figure 1 or equivalently, the true explained and unexplained components in (2) if $G$ and/or $X$ is confounded. We start by recalling the standard decomposition, where it is assumed that the observed outcome within a group is characterized by the linear equation

$$
Y^{g}=c^{g}+\sum_{k=1}^{K} X_{k}^{g} \beta_{k}^{g}+\epsilon^{g}, \quad g \in\{1,0\} .
$$

$Y^{g}$ and $X_{k}^{g}$ denote the conditional outcome and the conditional observed characteristics given $G=g \cdot{ }^{3}$ Likewise, $c^{g}$ and $\beta^{g}=$ $\left[\beta_{1}^{g}, \ldots, \beta_{K}^{g}\right]$ represent the constant and the slope coefficients, while $\epsilon^{g}$ denotes the error term, again conditional on the group variable. It is typically assumed (see, e.g., Fortin, Lemieux, and Firpo 2011) that

$$
E\left(\epsilon^{g} \mid X^{g}\right)=E(\epsilon \mid X, G=g)=0, \quad g \in\{1,0\},
$$

for which conditional independence of $\epsilon^{g}$ and $X^{g}$ is a sufficient condition (while $\epsilon$ denotes the unconditional error).

We assume that we have an iid sample drawn from $\{Y, X, G\}$ consisting of $n$ observations. Denote by $\bar{Y}^{g}$ and $\bar{X}^{g}$ the sample averages of $Y$ and $X$ conditional on $G=g$, with $\bar{X}^{g}=$ $\left[\bar{X}_{1}^{g}, \ldots, \bar{X}_{K}^{g}\right]$. The standard decomposition of Blinder (1973) and Oaxaca (1973) is given by

$$
\begin{aligned}
\bar{Y}^{1}-\bar{Y}^{0}= & \underbrace{\sum_{k=1}^{K}\left(\bar{X}_{k}^{1}-\bar{X}_{k}^{0}\right) \hat{\beta}_{k}^{1}}_{\text {explained component }(\hat{\psi})} \\
& +\underbrace{\left(\hat{c}^{1}-\hat{c}^{0}\right)+\sum_{k=1}^{K} \bar{X}_{k}^{0}\left(\hat{\beta}_{k}^{1}-\hat{\beta}_{k}^{0}\right)}_{\text {unexplained component }(\hat{\eta})} .
\end{aligned}
$$

$\hat{\beta}_{k}^{1}, \hat{\beta}_{k}^{0}$ denote the estimates of the coefficients $\beta_{k}^{1}, \beta_{k}^{0}$ and $\hat{\eta}, \hat{\psi}$ those of the unexplained and explained components, respectively.

If $G$ stands at the beginning of the causal mechanism affecting $Y$ as displayed in Figure 1, one can easily translate the standard decomposition into a simple mediation model. The latter consists of a system of linear equations characterizing each mediator as a function of $G$ and an error term $v$ and the outcome as a function of $G, X$, the interactions of $G$ and $X$, and an error term $\epsilon$ :

$$
\begin{gathered}
X_{k}=c_{X_{k}}+G \alpha_{k}+v_{k}, \quad \text { for } k \in\{1, \ldots, K\}, \\
Y=c_{Y}+G \delta+\sum_{k=1}^{K} X_{k} \theta_{k}+\sum_{k=1}^{K} G X_{k} \gamma_{k}+\epsilon .
\end{gathered}
$$

$c_{X_{k}}, \alpha_{k}, v_{k}$ denote the constant, coefficient, and error term in the equation of the $k$ th element in $X$. If applicable, we denote by the respective parameter without subscript $k$ the vector of $K$ elements, for example, $\alpha=\left[\alpha_{1}, \alpha_{2}, \ldots, \alpha_{K}\right] . c_{Y}, \delta, \theta$ represent the constant and the coefficients on $G$ and $X$ in the outcome equation, respectively, while $\gamma$ denotes the coefficients on the interactions. It is obvious that a causal interpretation of the various coefficients and the identification of direct and indirect effects generally requires that $v$ is not associated with $G$, and $\epsilon$ is not associated with $G$ and $X$. Therefore, this rather simple model rules out confounding of $G$ and/or $X$.

We now demonstrate that the indirect effect of $G$ on $Y$ working through $X$ in our linear mediation model (i.e., the effect of $G$ on $X$ times the effect of $X$ on $Y$ ) corresponds to the explained component in a wage decomposition. Imai, Keele, and Yamamoto (2010) provided the expression of the indirect effect for a scalar mediator $X_{k}$ when interaction between $G$ and $X_{k}$ is allowed for as in (7): $\alpha_{k}\left(\theta_{k}+G \gamma_{k}\right)$. It follows that the (overall) indirect effect through all mediators is the sum of the indirect

\footnotetext{
${ }^{3}$ Note the difference to Equation (1), which is defined in terms of potential outcomes $Y(g)$ rather than conditional outcomes $Y^{g}$.
} 
effects through each mediator:

$$
\text { (overall) indirect effect }=\sum_{k=1}^{K} \alpha_{k}\left(\theta_{k}+G \gamma_{k}\right) \text {. }
$$

Considering $X_{k}$, we note that in Equation (6), which contains a single binary regressor, the constant corresponds to the average mediator conditional on $G=0$, that is, $c_{X_{k}}=E\left(X_{k}^{0}\right)$, while $\alpha_{k}=E\left(X_{k}^{1}\right)-E\left(X_{k}^{0}\right)$. Furthermore, any coefficient $\theta_{k}$ reflects the effect of $X_{k}$ on $Y$ conditional on $G=0$ and is therefore equal to $\beta_{k}^{0}$ in (3). Correspondingly, the coefficient on the interaction term of $G$ and $X_{k}$ gives the difference in the effects of $X_{k}$ given $G=1$ and $G=0$, that is, $\gamma_{k}=\left(\beta_{k}^{1}-\beta_{k}^{0}\right)$. It follows that the indirect effect given in (8) conditional on $G=1$ is identical to the probability limit (plim) of the explained component $\hat{\psi}$ in $(5): \sum_{k=1}^{K} \alpha_{k}\left(\theta_{k}+G \gamma_{k}\right)=\sum_{k=1}^{K}\left[E\left(X_{k}^{1}\right)-E\left(X_{k}^{0}\right)\right]\left(\beta_{k}^{0}+\right.$ $\left.\left(\beta_{k}^{1}-\beta_{k}^{0}\right)\right)=\sum_{k=1}^{K}\left[E\left(X_{k}^{1}\right)-E\left(X_{k}^{0}\right)\right] \beta_{k}^{1}$.

Second, we show that the direct effect is equal to the unexplained component. Imai, Keele, and Yamamoto (2010) provided the expression for the direct effect under our linear mediation model:

$$
\text { direct effect }=\delta+\sum_{k=1}^{K} \gamma_{k}\left(c_{X_{k}}+\alpha_{k} G\right) .
$$

Note that $\delta$ reflects the part of the direct effect that is net of interactions with $X$. This obviously corresponds to the differences in the constants in Equation (3) for $G=1$ and $G=0$, which would be an alternative way to identify the effect of switching $G$ when holding $X$ fixed. Therefore, $\delta=c^{1}-c^{0}$. Conditional on $G=0$, (9) simplifies to $\delta+\sum_{k=1}^{K} c_{X_{k}} \gamma_{k}$. By recalling that $c_{X_{k}}=E\left(X_{k}^{0}\right)$ and $\gamma_{k}=\left(\beta_{k}^{1}-\beta_{k}^{0}\right)$, it follows that this corresponds to the plim of the unexplained component $\hat{\eta}$ in (5): $\delta+$ $\sum_{k=1}^{K} \gamma_{k}\left(c_{X_{k}}+\alpha_{k} G\right)=\left(c^{1}-c^{0}\right)+\sum_{k=1}^{K} E\left(X_{k}^{0}\right)\left(\beta_{k}^{1}-\beta_{k}^{0}\right)$.

Linking the standard decomposition to the mediation model highlights that the estimates $\hat{\psi}$ and $\hat{\eta}$ in (5) only bear a causal interpretation and converge to the true explained and unexplained components $\psi$ and $\eta$ in (2) as $n$ increases under the following conditions: (i) $G$ is exogenous, that is, not confounded by $v$, and (ii) $X$ is exogenous conditional on $G$, that is, not confounded by $\epsilon$ such that Equation (4) holds. Exogeneity of $G$ implies that the group variable is as good as randomly assigned w.r.t. $\epsilon$ (which enters $Y$ ) as well as any unobservables affecting $X$. This would for instance be violated if family background (e.g., parents' education and socio-economic status) was unobserved, entered $\epsilon$, and was also correlated with the group variable. Therefore, one might be particularly concerned if $G$ represents ethnicity, while gender is arguably randomly assigned by nature. However, even in the latter case, one could think of possible violations of exogeneity if for instance gender bias and thus, the gender ratio (through the inclination to get more children conditional on the gender of the first child) differed systematically across socioeconomic groups.

If $G$ is confounded by $\epsilon$, the conditional mean outcome $E\left(Y^{g}\right)=E(Y \mid G=g)$, which is the plim of $\bar{Y}^{g}$, does not correspond to the mean potential outcome $E(Y(g))$. Therefore, the left hand side of Equation (5) does not converge to $E(Y(1))-E(Y(0))$ such that not even the total causal effect of $G$ on $Y$ (the sum of the explained and unexplained compo- nents) is identified. Similarly, there might exist unobservables that jointly affect $G$ and $X$, which could even overlap with those in $\epsilon$ (e.g., family background drives educational choices). Then, $E\left(X^{g}\right)=E(X \mid G=g)$, the plim of $\bar{X}^{g}$, does not correspond to the mean potential mediator state $E(X(g))$. It follows that the right-hand side of (5) is asymptotically biased (i.e., does not correspond to the right-hand side of (2)) even if the left-hand side (i.e., the total effect) was identified.

We now consider the second issue that conditional exogeneity of $X$ given $G$ does not hold, implying that the effect of $X$ on $Y$ is confounded by $\epsilon$ even conditional on $G$. As in standard OLS models, this implies that the coefficients $\beta^{g}$ and $c^{g}$ in Equation (3) differ from the parameters $\beta(g)$ and $c(g)$ in the true causal model (1). Therefore, the right-hand side of (5) does not converge to the true decomposition (2) even if $G$ was exogenous. Figure 2 illustrates scenarios in which identification fails. In (a), $\epsilon$ jointly affects $G$ and $Y$ whereas in (b), the unobserved term $v$ jointly affects $G$ and $X$. Under both (a) and (b), exogeneity of $G$ does not hold. In (c), $\epsilon$ jointly affects $X$ and $Y$ (even conditional on $G$ ) such that (4) is violated. In applications, several issues might occur at the same time. Note that identification also fails if the respective unobserved terms do not directly influence $G$ and/or $X$, but are correlated with further unobservables that affect the group variable or the mediator, respectively. Furthermore, $\epsilon$ and $\nu$ might be correlated or even overlap.

Summing up, formulating the standard decomposition in terms of a mediation model facilitates understanding the assumptions required for causal inference, which are obviously not very attractive: $c_{X}$ and $\alpha$, and thus $E(X(0))$ and $E(X(1))-E(X(0))$, are only identified if $G$ is not confounded by $v$. Likewise, $\delta, \theta$, and $\gamma$ and thus, $c(1)-c(0), \beta(0)$, and $(\beta(1)-\beta(0))$ are only identified if $\epsilon$ does not confound $G$ and/or $X$. Otherwise, the unexplained and explained components in (5) are asymptotically biased and not to be causally interpreted. In addition, the standard decomposition imposes linearity and homogeneous effects within group states. Only if all these conditions do hold, the explained component corresponds to the indirect effect in a mediation model for $G=1$, for example, being male. It then reflects the wages differentials between males and females due to group-induced differences in the mediators $(X(1), X(0))$ assessed at the males' rate of returns to the mediators (e.g., the returns to education and occupation). In contrast, the unexplained component corresponds to the direct effect for $G=0$, for example, being female. It is the (potentially discriminatory) effect of gender on wage when holding the mediators fixed at their values among females, $X(0)$. As well acknowledged in the mediation literature, the direct and indirect effects defined on opposite group states add up to the total effect of $G$, namely $E(Y(1))-E(Y(0))$, the left-hand side of (2).

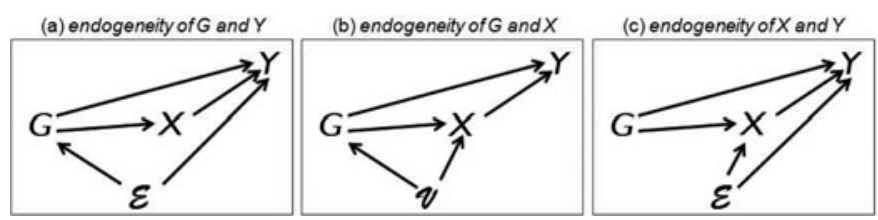

Figure 2. Scenarios in which identification fails. 
The question remains whether standard wage decompositions are useful at all in the arguably more realistic case of confounding, where the average observed differences $E\left(Y^{1}\right)-E\left(Y^{0}\right)$ and $E\left(X^{1}\right)-E\left(X^{0}\right)$ deviate from the average causal effects $E(Y(1)-Y(0))$ and $E(X(1)-X(0))$. While observed differences are descriptive about the status quo, they appear less suitable for deriving policy conclusions, which usually rely on causal inference based on comparing counterfactual states of the world. Basing wage decompositions on observed differences without controlling for confounders therefore gives explained and unexplained components that are hard to interpret. As elsewhere in econometrics, it is not obvious what to make of numbers obtained by conditioning on a battery of endogenous variables (in our case the mediators and the group variable). In this light, the terminology "explained component" may even appear misleading, because neither do endogenous group states causally explain observed differences in mediators, nor do endogenous group states and mediators causally explain observed differences in outcomes. One should also be cautious with interpreting the unexplained component as labor market discrimination, as often seen in the literature, because this would be a causal claim, too: namely, that a part of the supposedly causal effect of $G$ on wage which materializes in the observed wage gap is not driven by observed differences in the mediators, but by other (and unobserved) causal mechanisms like discrimination. As with the explained component, endogeneity jeopardizes the identification of the unexplained component based on standard decompositions. The latter may therefore not be very meaningful for deriving policy recommendations, as also argued by Kunze (2008).

\subsection{An Alternative Set of Identifying Assumptions}

We suggest the use of a different set of assumptions that extends standard decompositions in two dimensions: first, we allow for confounding of the group variable and the mediators, as long as it is related to observed covariates, which we denote by $W$. Second, we ease the linearity assumptions, which may entail misleading results due to functional form restrictions and inadequate extrapolation (see Barsky et al. 2002). Instead, we consider (at least for identification) a fully nonparametric model that maximizes flexibility in terms of model specification. To this end, we introduce a more general notation for the explained and unexplained components that comes from the nonparametric mediation literature (e.g., Pearl 2001; Robins 2003). First of all, note that instead of defining a potential outcome as a function of the group variable only, we may equivalently define it as a function of the group variable and the potential mediators on the causal pathway from the group to the outcome. In fact, $Y(g)=Y(g, X(g))$. This change in notation makes explicit that the potential outcome is affected by the group variable both directly and indirectly via $X(g)$. It allows us to rewrite the total effect of $G$ on $Y$ (the left-hand side of (2)) as $E(Y(1))-E(Y(0))=E[Y(1, X(1))]-E[Y(0, X(0))]$. More importantly, the latter expression can (by subtracting and adding $E[Y(1, X(0))]$ on the right-hand side) be disentangled into the indirect effect (or explained component) due to varying $X(1)$ and $X(0)$ while keeping the group fixed at $G=1$ and the direct effect (or unexplained component) of $G$ while keeping

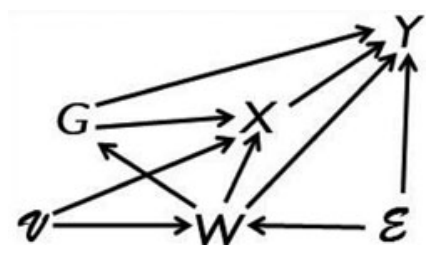

Figure 3. A causal framework in which sequential ignorability holds.

the mediators fixed at $X(0)$ :

$$
\begin{aligned}
E[Y(1, X(1))]-E[Y(0, X(0))] & =\underbrace{E[Y(1, X(1))]-E[Y(1, X(0))]}_{\psi} \\
& +\underbrace{E[Y(1, X(0))]-E[Y(0, X(0))]}_{\eta} .
\end{aligned}
$$

See also the discussion of these two components in Flores and Flores-Lagunes (2009), who derived an equivalent decomposition in their Equation (2).

Imai, Keele, and Yamamoto (2010) provided a sequential ignorability assumption under which $E[Y(1, X(1))]$, $E[Y(0, X(0))]$, and $E[Y(1, X(0))]$ are identified. ${ }^{4}$ Identify- $^{-}$ ing the latter parameter is particularly challenging because $Y(1, X(0))$ is never observed.

Assumption 1 (sequential ignorability).

(a) $\left\{Y\left(g^{\prime}, x\right), X(g)\right\} \perp G \mid W$ for all $g^{\prime}, g \in\{0,1\}$ and $x$ in the support of $X$,

(b) $Y\left(g^{\prime}, x\right) \perp X \mid G=g, W=w$ for all $g^{\prime}, g \in\{0,1\}$ and $x, w$ in the support of $X, W$,

(c) $\operatorname{Pr}(G=g \mid X=x, W=w)>0$ for all $g \in\{0,1\}$ and $x, w$ in the support of $X, W$.

By Assumption 1(a), $G$ is conditionally independent of the mediators and of any unobservable factors jointly affecting the group status on the one hand and the mediator and/or the outcome on the other hand, given the observed confounders $W$. Assumption 1(b) imposes conditional independence of the mediator given the confounders and the group status. That is, conditional on $G$ and $W$, the effect of the mediator on the outcome is assumed to be unconfounded. Assumption 1(c) is a common support restriction requiring that the conditional probability to be in a particular group given $X$ and $W$ (henceforth referred to as propensity score) is larger than zero. Note that by Bayes' theorem, Assumption 1(c) equivalently implies that $\operatorname{Pr}(X=x \mid G=$ $g, W=w$ ) $>0$ (or in the case of $X$ being continuous, that the conditional density of $X$ given $G$ and $W$ is larger than zero: $\left.f_{X \mid G, W}(x, g, w)>0\right)$. That is, conditional on $W$, the mediator state must not be a deterministic function of the group, otherwise identification is infeasible due to a lack of comparable units in terms of $X$ across groups. Furthermore, from Assumption 1(c) it also follows that $\operatorname{Pr}(G=g \mid W=w)>0$ for all $g \in\{0,1\}$ and $w$ in the support of $W$, which must hold to find comparable units in terms of the confounders $W$. Figure 3 displays a causal framework that is in line with sequential ignorability.

\footnotetext{
${ }^{4}$ Equivalent or closely related assumptions were considered in Pearl (2001), Flores and Flores-Lagunes (2009), Imai, Keele, and Yamamoto (2010), and Tchetgen Tchetgen, and Shpitser (2012), among others.
} 
The following linear model satisfies the sequential ignorability assumption if $v$ is conditionally independent of $G$ given $W$ and $\epsilon$ is conditionally independent of $G$ and $X$ given $W$. Note that this parametric specification does not require the common support Assumption 1(c) due to the possibility to extrapolate beyond observed data, which, however, comes at the cost of considerably stronger functional form assumptions than needed for nonparametric identification:

$$
\begin{gathered}
X_{k}=c_{X_{k}}+G \alpha_{k}+\sum_{j=1}^{J} W_{j} \omega_{j k}+v_{k}, \quad \text { for } \quad k \in\{1, \ldots, K\} \\
Y=c_{Y}+G \delta+\sum_{k=1}^{K} X_{k} \theta_{k}+\sum_{k=1}^{K} G X_{k} \gamma_{k}+\sum_{j=1}^{J} W_{j} \lambda_{j k}+\epsilon .
\end{gathered}
$$

Given that the conditional independence assumptions are satisfied, we can in principle allow for a much more flexible nonparametric model when identifying the explained and unexplained components:

$$
\begin{aligned}
X_{k} & =\chi_{k}(G, W, \nu), \quad \text { for } k \in\{1, \ldots, K\} \\
Y & =\phi(G, X, W, \epsilon),
\end{aligned}
$$

$\phi$ and $\chi$ are general functions that remain unspecified by the researcher, so that linearity and homogeneous effects within group states need not be assumed as it is the case in the standard decomposition. Note that the potential outcome notation can be readily translated into this nonparametric model: $E\left[Y\left(g, X\left(g^{\prime}\right)\right)\right]=$ $E\left[\phi\left(g, X\left(g^{\prime}\right), W, \epsilon\right)\right]=E\left[\phi\left(g, \chi_{k}\left(g^{\prime}, W, \nu\right), W, \epsilon\right)\right]$ for $g, g^{\prime} \in$ $\{1,0\}$.

Under Assumption 1, Propositions 1 and 2 in Huber (2013) for the identification of direct and indirect effects can be used to nonparametrically identify the explained and unexplained components based on reweighting observations by the inverse of the propensity scores $\operatorname{Pr}(G=1 \mid X, W)$ and $\operatorname{Pr}(G=1 \mid W)$ :

$$
\begin{aligned}
\psi= & E\left[\frac{Y \cdot G}{\operatorname{Pr}(G=1 \mid W)}\right] \\
& -E\left[\frac{Y \cdot G}{\operatorname{Pr}(G=1 \mid X, W)} \cdot \frac{1-\operatorname{Pr}(G=1 \mid X, W)}{1-\operatorname{Pr}(G=1 \mid W)}\right], \\
\eta= & E\left[\frac{Y \cdot G}{\operatorname{Pr}(G=1 \mid X, W)} \cdot \frac{1-\operatorname{Pr}(G=1 \mid X, W)}{1-\operatorname{Pr}(G=1 \mid W)}\right] \\
& -E\left[\frac{Y \cdot(1-G)}{1-\operatorname{Pr}(G=1 \mid W)}\right] .
\end{aligned}
$$

The proof of this result is provided in Appendix A. $E\left[\frac{Y \cdot G}{\operatorname{Pr}(G=1 \mid W)}\right]$ and $E\left[\frac{Y \cdot(1-G)}{1-\operatorname{Pr}(G=1 \mid W)}\right]$ correspond to the mean potential outcomes $E(Y(1))$ and $E(Y(0))$, because weighting by the inverse of $\operatorname{Pr}(G=1 \mid W)$ and $1-\operatorname{Pr}(G=1 \mid W)$, respectively, adjusts the distribution of confounders $W$ given $G=1,0$ to that in the total population. $E\left[\frac{Y \cdot G}{\operatorname{Pr}(G=|| X, W)} \cdot \frac{1-\operatorname{Pr}(G=|| X, W)}{1-\operatorname{Pr}(G=|| W)}\right]$, on the other hand, identifies $E[Y(1, X(0))]$, because the law of iterated expectations and Bayes' theorem imply that $\frac{1-\operatorname{Pr}(G=1 \mid X, W)}{\operatorname{Pr}(G=1 \mid X, W) \cdot(1-\operatorname{Pr}(G=1 \mid W)}$ reweights $W, X$ given $G=1$ to the distribution of $W, X(0)$ in the total population.

The attractiveness of expressions (15) and (16) is that they can be straightforwardly estimated by their sample counter- parts with (parametric or nonparametric) plug-in estimators for the propensity scores $\operatorname{Pr}(G=1 \mid X, W)$ and $\operatorname{Pr}(G=1 \mid W)$. However, estimation based on weighting also has its drawbacks: if the common support assumption 1(c) is close to being violated, estimation may be unstable due to an explosion of the variance, see for instance (Khan and Tamer 2010). Furthermore, weighting may be less robust to propensity score misspecification than other classes of estimators, as documented for instance in Kang and Schafer (2007) and Waernbaum (2012). Alternative non- or semiparametric estimators of direct and indirect effects include the regression estimator of Imai, Keele, and Yamamoto (2010) and "multiply robust" estimation either based on the efficient influence function, see Tchetgen Tchetgen, and Shpitser (2012), or on targeted maximum likelihood, see Zheng and van der Laan (2012).

Note that if $G$ and $X$ were not confounded by $W$, Assumption 1(a) could be replaced by $\left\{Y\left(g^{\prime}, x\right), X(g)\right\} \perp G$ (random assignment of $G$ ) and 1 (b) by $Y\left(g^{\prime}, x\right) \perp X \mid G=g$. This would correspond to a nonparametric version of the model described in (6) and (7). It is easy to show that in this case the identification results (15) and (16) simplify to

$$
\begin{aligned}
\psi=E & {\left[\frac{Y \cdot G}{\operatorname{Pr}(G=1)}\right] } \\
& -E\left[\frac{Y \cdot G}{\operatorname{Pr}(G=1 \mid X)} \cdot \frac{1-\operatorname{Pr}(G=1 \mid X)}{1-\operatorname{Pr}(G=1)}\right], \\
\eta= & E\left[\frac{Y \cdot G}{\operatorname{Pr}(G=1 \mid X)} \cdot \frac{1-\operatorname{Pr}(G=1 \mid X)}{1-\operatorname{Pr}(G=1)}\right] \\
& -E\left[\frac{Y \cdot(1-G)}{1-\operatorname{Pr}(G=1)}\right] .
\end{aligned}
$$

Interestingly, (18) looks identical to the identification of the average treatment effect on the nontreated in Hirano, Imbens, and Ridder (2003) based on weighting, however, under the conceptually different framework of a conditionally independent treatment given observed pretreatment (or pregroup) variables $X$. The crucial difference is that in Hirano, Imbens, and Ridder (2003), conditioning on $X$ controls for selection into the treatment (or group), whereas here, with $X$ being a post-group mediator, conditioning on $X$ controls for the indirect effect via $X$ to identify the unexplained component. Obviously, this is only feasible if $G$ and $X$ are not confounded. In this case, any nonparametric method for the average treatment effect on the nontreated under conditionally independent treatment assignment consistently estimates $\eta$, including matching, see Rubin (1974), and doubly robust estimation, see Rothe and Firpo (2013). However, ruling out any confounding appears restrictive in empirical applications.

Before concluding this section, it has to be pointed out that even though Assumption 1 improves on the identifying assumptions implied by the standard decomposition by permitting observed confounders of $G$ and $X$, it is still quite restrictive. In particular, it does not allow for post-group confounders of $X$ which are affected by $G$, which would make identification more cumbersome. As discussed in the empirical application in Section 4, it may, however, appear plausible that some variables influencing both the mediators and the outcome are themselves influenced by the group state (e.g., the development of 
personality traits as a function of gender/ethnicity). We therefore briefly review alternative identification strategies suggested in Robins and Richardson (2010), Albert and Nelson (2011), Tchetgen Tchetgen and VanderWeele (2012), Imai and Yamamoto (2013), and Huber (2013) which do allow for post-group confounders that are a function of $G$ based on various sets of assumptions. A crucial difference to Assumption 1 is that in addition to particular sequential ignorability assumptions, further restrictions on the group-mediator, confounder-mediator, or mediator-outcome relation are required for identification. Imai and Yamamoto (2013), for instance, assumed that the individual group-mediator-interaction effects are homogeneous (i.e., the same for all individuals with the same values in $X$ ). Assumption 5 in Huber (2013) restricts the average group-mediatorinteraction effects to be homogeneous conditional on the pre and post-group confounders and assumes the outcome to be linear in the mediators. Alternatively, Albert and Nelson (2011) and Robins and Richardson (2010) acknowledge that the parameters of interest are identified if the potential values of the post-group confounders under $G=1$ and $G=0$ are either independent of each other or the functional form of their dependence is known (which appears, however, unrealistic in applications). Finally, Tchetgen Tchetgen and VanderWeele (2012) showed that identification is feasible (under particular ignorability conditions) if all post-group confounders are binary and monotonic in $G$ or if there are no average interaction effects of the postgroup confounders and the mediators on the outcome (which is testable in the data). ${ }^{5}$

\section{SIMULATION}

This section presents a brief simulation study in which the following data-generating process (DGP) is considered:

$$
\begin{aligned}
G & =I\{\alpha W+\xi>0\}, \\
X & =0.5 G+\beta W+\nu, \\
Y & =G+X+G X+\beta W+\gamma X W+\epsilon, \\
W, \xi, \nu, \epsilon & \sim N(0,1), \text { independently of each other. }
\end{aligned}
$$

$\xi, \nu, \epsilon$ are the error terms, while $W$ is a potential confounder. For $\beta \neq 0$, the mediator is confounded, for $\alpha, \beta \neq 0$ both the group and the mediator are confounded. Finally, if $\gamma \neq 0$, then there exists an interaction of $X$ and $W$ in the outcome equation. The explained component is $\psi=E[0.5(1+G X) \mid G=1]=$ $0.5+E[X \mid G=1]=1$, while the unexplained component is $\eta=E[1+(0+0.5 G) \mid G=0]=1$. We simulate 1000 times for various choices of $\alpha, \beta, \gamma$ and two sample sizes $(n=$

\footnotetext{
${ }^{5}$ As elsewhere in empirical economics, it may not appear plausible that all (pre- or post-group) confounders are observed. If credible instrumental variables for the group and/or the mediators are available, they may provide an alternative source for identifying direct and indirect effects, see for instance the nonparametric approaches in Imai, Tingley, and Yamamoto (2012) and Yamamoto (2013). If no instruments are at hand and confounders are not observed, partial identification based on deriving upper and lower bounds on the direct and indirect effects might nevertheless be informative, see for instance (Cai et al. 2008; Flores and Flores-Lagunes 2010). However, most of these instrumentand bounds-based approaches were developed for the case of a scalar mediator and may not easily generalize to the framework of multiple mediators as usually encountered in wage decompositions.
}

500, 2000), considering three different estimators: the standard decomposition ("decomposition") of Blinder (1973) and Oaxaca (1973) based on (5), OLS estimation of direct and indirect effects ("mediation.ols"), which controls for the confounder $W$ based on the linear Equations (11) and (12), and finally, semiparametric inverse probability weighting (IPW) based on the sample analogs of (15) and (16), respectively ("mediation.ipw"). The propensity scores $\operatorname{Pr}(G=1 \mid X, W)$ and $\operatorname{Pr}(G=$ $1 \mid W)$ entering the IPW formulas are estimated by the covariatebalancing propensity score method of Imai and Ratkovic (2014), which models group assignment while at the same time optimizing the covariate balance using an empirical likelihood approach. ${ }^{6}$

Table 1 presents the bias, standard deviation (s.d.), and root mean squared error (RMSE) of the various estimators. For $\alpha=0, \beta=0, \gamma=0, W$ does neither confound $X$, nor $G$ and all methods are consistent and close to being unbiased. Not unexpectedly, semiparametric IPW has a somewhat higher standard deviation than the parametric methods due to imposing weaker assumptions. Setting $\beta=1$ in the second panel of Table 1 introduces confounding of the mediator. Now, the standard decomposition is biased and has a substantially higher RMSE than the other two methods which control for $W$. In the third panel, $\alpha=0.25, \beta=1, \gamma=0$, which implies that also $G$ is confounded. This further increases the bias of the standard decomposition, while the other estimators are again almost unbiased. In the fourth panel, $\alpha=0.25, \beta=1, \gamma=0.5$, such that there exists an interaction of $W$ and $X$. As the OLS estimator does not account for the latter (but only for $W$ ), it is somewhat biased due to this misspecification. In contrast, IPW remains close to being unbiased due to its higher flexibility w.r.t. structural form assumptions and now dominates the other methods in terms of bias and RMSE. Summing up, the simulations demonstrate the nonrobustness of the standard decomposition to confounding and violations of functional form restrictions as well as the potential gains coming from the use of more flexible estimation methods that are based on less rigid identifying assumptions.

\section{EMPIRICAL APPLICATION}

In this section, we provide an empirical illustration in which we investigate the robustness of the results when applying various decomposition methods to the ethnic wage gap among males participating in the National Longitudinal Survey of Youth 1979 (NLSY79). The NLSY79 was designed to represent the youth population in the U.S. in 1979 and consists of individuals that were between 14 and 22 years old in that year. It was repeated annually through 1994 and every other year ever after. The survey contains very rich information on labor market relevant characteristics such as education, detailed work experience,

\footnotetext{
${ }^{6}$ IPW based on probit models for the propensity scores was also considered in the simulations, but the results are not reported because it behaved very similarly to IPW based on the empirical likelihood approach of Imai and Ratkovic (2014). Note that semiparametric IPW estimation using parametric propensity score models is $\sqrt{n}$-consistent, which can be shown in a sequential GMM framework (see Newey 1984) in which propensity score estimation represents the first step and the IPW decomposition the second step.
} 
Table 1. Simulations

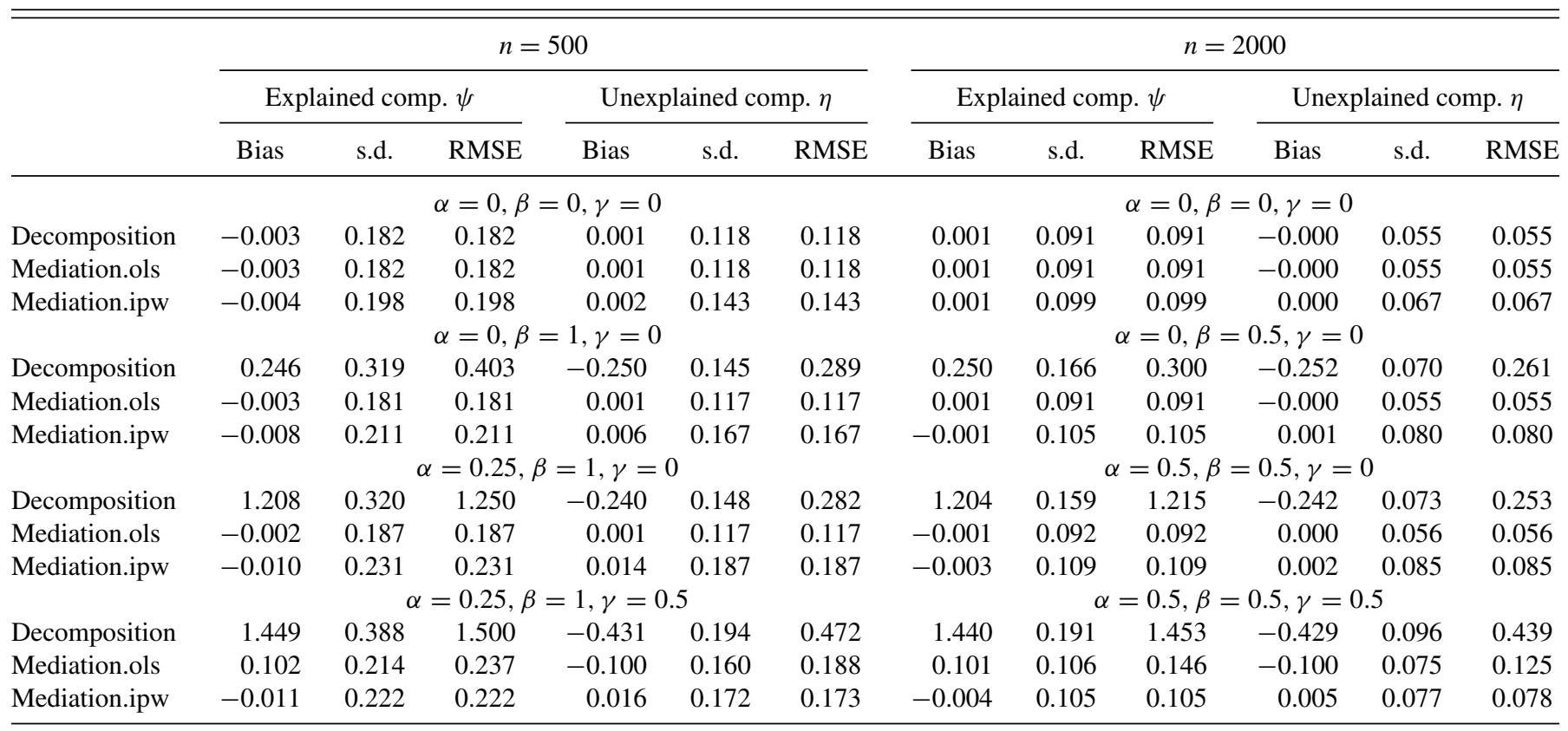

NOTE: "Decomposition" refers to estimation based on (5), "mediation.ols" to OLS estimation of (11) and (12), and "mediation.ipw" to weighting based on (15) and (16), where $\operatorname{Pr}(G=g \mid X, W)$ and $\operatorname{Pr}(G=g \mid W)$ are estimated by the (Imai and Ratkovic 2014) procedure.

occupational choices, and many others. Our analysis focuses on males only, for whom the literature typically finds larger ethnic wage differentials than for females, see, for instance, Hellerstein, Neumark, and Troske (1999) or O'Neill and O'Neill (2005). To be precise, we confine our sample to non-Hispanic males to estimate the decomposition for blacks $(G=0)$ and whites $(G=1)$.

The outcome of interest $(Y)$ is the log hourly wage in the year 2000 of the then $35-43$ years old survey participants, which is observed for 2571 cases or $48 \%$ of the initial non-Hispanic male sample, thereof 851 blacks and 1720 whites. Similarly to what is standard in the literature, our variables $X$ characterizing the explained component include age, education, labor market history, tenure with the current employer, industry, type of occupation, whether living in an urban area, and region. Table 2 provides descriptive statistics on these variables, namely the respective mean values for blacks and whites, the mean differences, and the $t$-values ( $t$-val) and $p$-values ( $p$-val) based on two-sample $t$-tests.

Table 2. Means and mean differences in mediators across blacks and whites

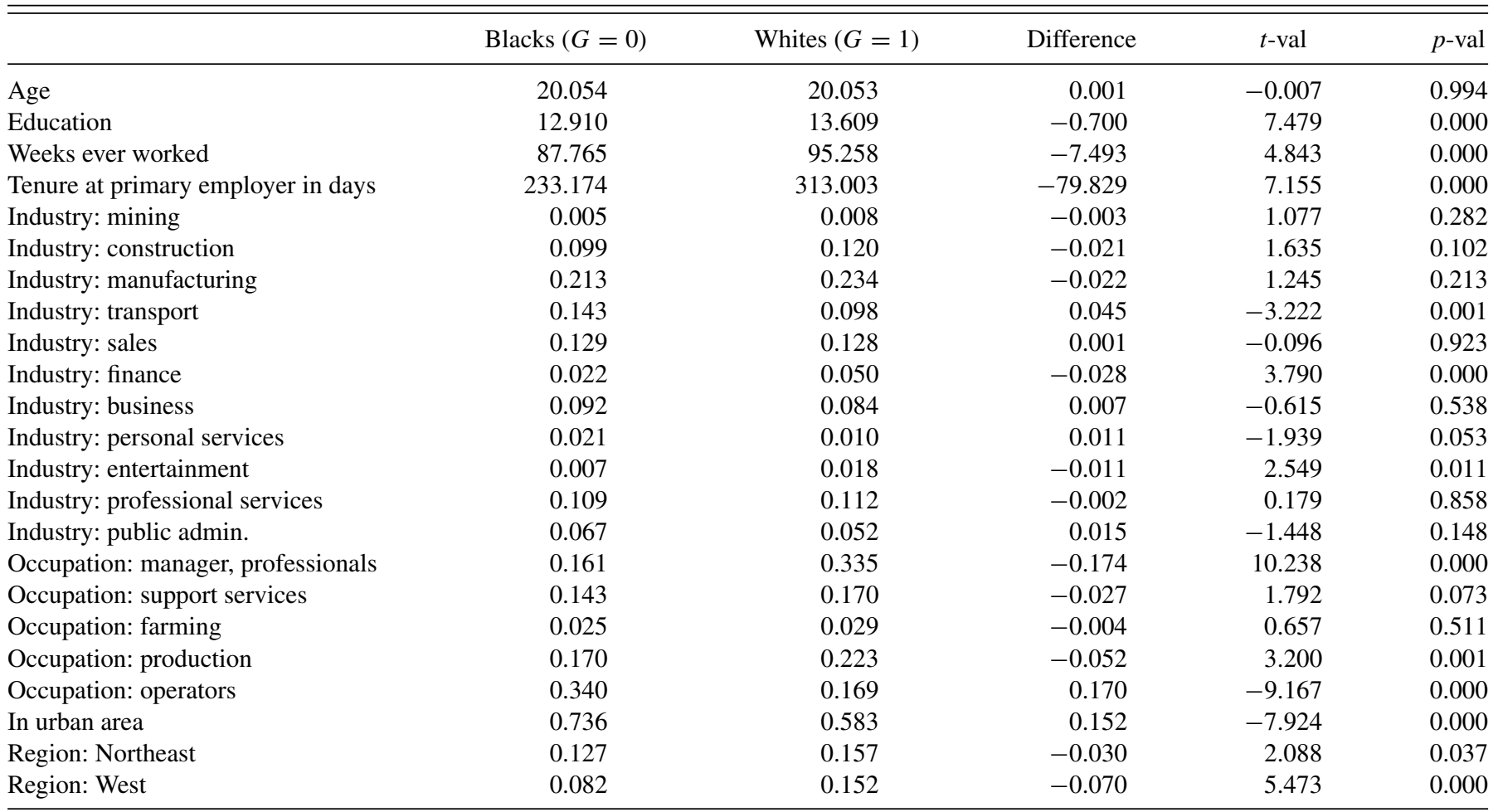


Table 3. Black-White wage gap decomposition based on NLSY79

\begin{tabular}{|c|c|c|c|c|c|c|c|c|c|c|c|}
\hline & \multicolumn{3}{|c|}{ Total gap in log wages } & \multicolumn{4}{|c|}{ Explained component $\hat{\psi}$} & \multicolumn{4}{|c|}{ Unexplained component $\hat{\eta}$} \\
\hline & Estimate & s.e. & $p$-val & Estimate & s.e. & $p$-val & $\%$ of total & Estimate & s.e. & $p$-val & $\%$ of total \\
\hline Ipw & 0.434 & 0.044 & 0.000 & 0.306 & 0.041 & 0.000 & 70.4 & 0.128 & 0.057 & 0.024 & 29.6 \\
\hline Mediation.ols & 0.288 & 0.048 & 0.000 & 0.176 & 0.033 & 0.000 & 61.3 & 0.112 & 0.053 & 0.036 & 38.7 \\
\hline Mediation.ipw & 0.280 & 0.046 & 0.000 & 0.171 & 0.060 & 0.004 & 60.9 & 0.109 & 0.069 & 0.114 & 39.1 \\
\hline
\end{tabular}

NOTE: "Decomposition" refers to estimation based on (5), "ipw" to weighting based on (17) and (18) not controlling for W, "mediation.ols" to OLS estimation of (11) and (12) which controls for $W$, and "mediation.ipw" to weighting based on (15) and (16) which also controls for $W$. All standard errors (s.e.) are based on 999 bootstrap replications.

We see that the groups differ significantly in terms of labor market experience, job characteristics, and region. As mentioned before, conventional decompositions would merely make use of $X$ without conditioning on potential confounders of $X$ and/or $G$. Here, we also consider controlling for pregroup variables $W$ that reflect family background and could potentially confound the group variable and the mediators. To be specific, $W$ contains mother's and father's levels of education as well as dummies indicating whether the respondent or her/his mother were born in a foreign country.

Table 3 provides the estimates of the total wage gap as well as the explained and unexplained components using four different decomposition methods along with standard errors (s.e.) and $p$ values (p-val) based on 999 bootstrap replications. It also gives the explained and unexplained components as percentages of the total gap ( $\%$ of total) to assess their relative importance. The total gap in average log wages of blacks and whites is $0.434 \mathrm{log}$ points. The standard decomposition of Blinder (1973) and Oaxaca (1973) ("decomposition") suggests that $0.299 \log$ points or $68.9 \%$ of the total gap are explained by differences in $X$, while $0.135 \log$ points or $31.1 \%$ are unexplained. Both estimates are highly significant. We also consider the IPW estimator ("ipw") based on (17) and (18), which is more flexible in terms of functional form assumptions but does not control for $W$ either. As in the simulations, $\operatorname{Pr}(G=1 \mid X)$ is estimated by the empirical likelihood approach of Imai and Ratkovic (2014). The propensity score specification is provided in Table 4 in Appendix A.2. As this semiparametric method does not extrapolate to sparse data regions, its applicability hinges on a sufficiently high overlap of the propensity scores across groups, also known as common support. Figures 4 and 5 in Appendix A.3 provides density estimates of the within-group propensity scores using the logspline command in R. The common support appears decent, so that for any individual comparable observations w.r.t. the propensity score can be found in the respective other group. We see that despite the more flexible functional form assumptions, the results based on weighting (without controlling for $W$ ) are very similar to those of the standard decomposition.

We also consider two estimators controlling for family background characteristics $W$, namely OLS estimation of direct and indirect effects ("mediation.ols") based on Equations (11) and (12), and IPW ("mediation.ipw") based on (15) and (16). The propensity score specification of $\operatorname{Pr}(G=1 \mid X, W)$ is provided in Appendix A.2 and the common support, which is again quite satisfactory, is displayed in Appendix A.3. ${ }^{7}$ Note that the total

\footnotetext{
${ }^{7} \mathrm{We}$ also investigated whether the Imai and Ratkovic (2014)-based estimate of $\operatorname{Pr}(G=1 \mid X, W)$ does indeed optimally balance the distributions of $X, W$
}

gap now corresponds to the difference in log wages of blacks and whites after making both groups comparable in $W$. It is considerably smaller than the (initial) wage gap ignoring confounding by $W(0.434)$, no matter whether estimation is based on OLS $(0.288)$ or IPW $(0.280)$. This suggests that the total impact of ethnicity on wages is overestimated when not controlling for family background. The same applies to the magnitudes of explained components, which drop substantially when controlling for $W$ (from roughly 0.3 to less than $0.18 \log$ points). The unexplained components, in contrast, decrease only moderately to $0.11 \mathrm{log}$ points. Therefore, their relative importance measured as percentage of the total gap increases somewhat to $39 \%$. As before, the OLS and IPW estimates are very similar, with the latter being somewhat nosier because of weaker functional form assumptions. In particular, the unexplained component based on IPW is not significantly different from zero at the $10 \%$ level, while that based on OLS is significant at the 5\% level.

Our results suggest that the decomposition estimates are not robust to ignoring potential pre-group confounders, but may entail overestimation of the absolute values of the explained and unexplained components as well as the total wage gap. However, even the estimates based on controlling for $W$ should not be taken at face value. First, in addition to the family background variables considered, there might exist further pre-group confounders that were omitted in the estimation. Potential examples include parents' personality traits, parents' health, and neighborhood conditions, which might differ systemically across $G$ or $X$ and at the same time affect labor market success later in life, entailing a violation of Assumption 1. Second, as a further issue not considered in our analysis, there may also exist post-group confounders of $X$ that are themselves potentially affected by $G$. This case is ruled out in Assumption 1, but clearly a plausible scenario, given that the mediators are measured with a substantial time lag after the determination of $G$ (at birth).

As an example, consider the possibility that $G$ affects the development of personality traits (e.g., through a systematically different exposure to discrimination, peer groups, etc., while growing up), which themselves influence $X$ (e.g., schooling and occupation) as well as potential wages. Further possible sources of post-group confounding are attrition and selection into employment, as roughly half of the initial sample had dropped out

across groups as one would expect from empirical likelihood estimation. In the original data prior to IPW weighting, the mean and maximum absolute differences in $X, W$ across groups amount to 1.698 and 79.828 , respectively. The standardized mean and maximum absolute differences (standardized by the standard deviations of the respective variables) are 0.142 and 0.592 . After IPW weighting, any of the measures is 0.000 , so that $X, W$ have been balanced successfully. 
of the survey by 2000 or did not report any employment. ${ }^{8}$ Therefore, controlling for pre-birth confounders does likely not suffice for tackling mediator endogeneity. At the very least, our analysis and the sensitivity of the results to different sets of assumptions demonstrate the importance of considering the problem of confounding in wage decompositions, an issue apparently neglected in much of the literature. Future research may discuss the implementation and attractiveness of alternative identification strategies that also allow for post-group confounders (see, for instance, the work of Robins and Richardson 2010, Albert and Nelson 2011, Imai and Yamamoto 2013, Huber 2013, and Tchetgen Tchetgen and VanderWeele 2012 mentioned in Section 2.3) in the context of wage decompositions.

\section{CONCLUSION}

This article has made explicit the identifying assumptions underlying conventional decompositions of gender or ethnic wage gaps, which continue to receive much attention in the empirical labor literature, by translating the decomposition into an equivalent model for mediation analysis. Inspecting the latter immediately shows that conventional decompositions do not control for confounders of the group variable (e.g., gender or ethnicity) and/or the variables characterizing the explained component (e.g., education and occupation), if the latter are determined after the group variable. This appears to be the standard case, as gender or ethnicity are determined at or prior to birth and therefore precede mediators like education or profession. For this reason, we have suggested the use of an alternative set of identifying assumptions that assumes exogeneity of the group variable and the mediators only conditional on observed confounders. Then, the unexplained and explained components of the decomposition can be nonparametrically identified by using a simple weighting expression that reweights observations by the inverse of the conditional propensity to belong to a particular group given the mediators and confounders. Finally, we have provided a simulation study as well as an empirical application to data from the National Longitudinal Survey of Youth 1979, in which we have also pointed to approaches permitting to further relax our identifying assumptions.

\section{A. APPENDIX}

\section{A.1 Proof of Equations (15) and (16) under Assumption 1}

The following proof is closely related to Huber (2013). To prove Equations (15) and (16) under Assumption 1, we need to show that $E[Y(g, X(g))]$ for $g \in\{1,0\}$ and $E[Y(1, X(0))]$ are identified. Starting with the former, note that

$$
\begin{aligned}
E[Y(g, X(g))] & =\int E[Y(g, X(g)) \mid W=w] d F_{W}(w) \\
& =\int E[Y \mid G=g, W=w] d F_{W}(w)
\end{aligned}
$$

${ }^{8}$ We refer to MaCurdy, Mroz, and Gritz (1998) for a detailed analysis of the attrition patterns in the NLSY79.

$$
\begin{aligned}
& =E\left[E\left[\frac{Y \cdot I\{G=g\}}{\operatorname{Pr}(G=g \mid W)} \mid W=w\right]\right] \\
& =E\left[\frac{Y \cdot I\{G=g\}}{\operatorname{Pr}(G=g \mid W)}\right] .
\end{aligned}
$$

The first equality follows from the law of iterated expectations and from replacing the expectation by an integral, the second from Assumption 1 (a), the third from basic probability theory and from replacing the integral by the expectation, and the last from the law of iterated expectations.

Concerning the latter,

$$
\begin{aligned}
& E[Y(1, X(0))] \\
= & \iint E[Y(1, x) \mid X(0)=x, W=w] d F_{X(0) \mid W=w}(x) d F_{W}(w) \\
= & \iint E[Y(1, x) \mid G=1, X=x, W=w] \\
& \times d F_{X \mid G=0, W=w}(x) d F_{W}(w) \\
= & \iint E[Y \mid G=1, X=x, W=w] \cdot \frac{\operatorname{Pr}(G=0 \mid X, W)}{\operatorname{Pr}(G=0 \mid W)} \\
& \times d F_{X \mid W=w}(x) d F_{W}(w) \\
= & E\left[E \left[E\left[\frac{Y \cdot G}{\operatorname{Pr}(G=1 \mid X, W)} \mid X=x, W=w\right]\right.\right. \\
& \left.\left.\times \frac{1-\operatorname{Pr}(G=1 \mid X, W)}{1-\operatorname{Pr}(G=1 \mid W)} \mid W=w\right]\right] \\
= & E\left[\frac{Y \cdot G}{\operatorname{Pr}(G=1 \mid X, W)} \cdot \frac{1-\operatorname{Pr}(G=1 \mid X, W)}{1-\operatorname{Pr}(G=1 \mid W)}\right]
\end{aligned}
$$

The first equality follows from the law of iterated expectations and from replacing the outer expectations by integrals, the second from Assumptions 1(a) and 1(b), the third from Bayes' theorem, the fourth from basic probability theory and from replacing the integrals by expectations, and the last from the law of iterated expectations.

Therefore, the explained component $\psi=E[Y(1, X(1))]-$ $E[Y(1, X(0))]$ is equal to

$$
\begin{aligned}
E\left[\frac{Y \cdot G}{\operatorname{Pr}(G=1 \mid W)}\right]-E\left[\frac{Y \cdot G}{\operatorname{Pr}(G=1 \mid X, W)}\right. \\
\left.\times \frac{1-\operatorname{Pr}(G=1 \mid X, W)}{1-\operatorname{Pr}(G=1 \mid W)}\right],
\end{aligned}
$$

while the unexplained component $\eta=E[Y(1, X(0))]-E[Y(0, X(0))]$ is equal to

$$
\begin{array}{r}
E\left[\frac{Y \cdot G}{\operatorname{Pr}(G=1 \mid X, W)} \cdot \frac{1-\operatorname{Pr}(G=1 \mid X, W)}{1-\operatorname{Pr}(G=1 \mid W)}\right] \\
-E\left[\frac{Y \cdot(1-G)}{1-\operatorname{Pr}(G=1 \mid W)}\right] .
\end{array}
$$


Table 4. Propensity score specifications based on the Imai and Ratkovic (2014) procedure

\begin{tabular}{|c|c|c|c|c|c|c|c|c|}
\hline & \multicolumn{4}{|c|}{$\operatorname{Pr}(G=1 \mid X, W)$} & \multicolumn{4}{|c|}{$\operatorname{Pr}(G=1 \mid X)$} \\
\hline$X$ & & & & & & & & \\
\hline Age & -0.047 & 0.019 & -2.418 & 0.016 & -0.051 & 0.023 & -2.245 & 0.025 \\
\hline Education: 12 yrs & -0.421 & 0.289 & -1.455 & 0.146 & -0.061 & 0.194 & -0.314 & 0.754 \\
\hline Education: 13 yrs & -0.815 & 0.373 & -2.185 & 0.029 & -0.074 & 0.329 & -0.224 & 0.823 \\
\hline Education: 16 yrs & -0.634 & 0.375 & -1.689 & 0.091 & 0.329 & 0.226 & 1.458 & 0.145 \\
\hline Education: 17 yrs & -0.820 & 0.544 & -1.508 & 0.131 & 0.195 & 0.565 & 0.344 & 0.731 \\
\hline Education: 18 yrs or more & -0.321 & 0.447 & -0.717 & 0.473 & 0.767 & 0.353 & 2.174 & 0.030 \\
\hline Weeks ever worked & 0.005 & 0.002 & 2.546 & 0.011 & 0.003 & 0.003 & 1.040 & 0.298 \\
\hline Weeks ever worked missing & -0.687 & 0.533 & -1.289 & 0.197 & -0.599 & 0.442 & -1.356 & 0.175 \\
\hline Years ever worked & 0.122 & 0.018 & 6.947 & 0.000 & 0.137 & 0.017 & 8.211 & 0.000 \\
\hline Industry: construction & -0.166 & 0.223 & -0.746 & 0.456 & -0.254 & 0.229 & -1.106 & 0.269 \\
\hline Industry: manufact. durables & -0.488 & 0.253 & -1.930 & 0.054 & -0.586 & 0.251 & -2.339 & 0.019 \\
\hline Industry: manufact. non-dur. & -0.310 & 0.203 & -1.523 & 0.128 & -0.285 & 0.229 & -1.244 & 0.213 \\
\hline Industry: transport & -0.829 & 0.201 & -4.130 & 0.000 & -0.907 & 0.190 & -4.780 & 0.000 \\
\hline Industry: wholesale & -0.468 & 0.540 & -0.866 & 0.386 & -0.197 & 0.398 & -0.494 & 0.621 \\
\hline Industry: retail & -0.479 & 0.204 & -2.347 & 0.019 & -0.579 & 0.247 & -2.340 & 0.019 \\
\hline Industry: finance & -0.171 & 0.389 & -0.440 & 0.660 & -0.293 & 0.274 & -1.068 & 0.286 \\
\hline Industry: business & -0.399 & 0.211 & -1.893 & 0.058 & -0.400 & 0.210 & -1.904 & 0.057 \\
\hline Industry: personal services & -0.891 & 0.436 & -2.044 & 0.041 & -1.009 & 0.547 & -1.843 & 0.065 \\
\hline Industry: entertainment & 1.112 & 0.523 & 2.127 & 0.033 & 0.651 & 0.402 & 1.618 & 0.106 \\
\hline Industry: professional services & -0.651 & 0.256 & -2.542 & 0.011 & -0.816 & 0.222 & -3.671 & 0.000 \\
\hline Urban area missing & 0.301 & 1.105 & 0.273 & 0.785 & 0.557 & 0.701 & 0.794 & 0.427 \\
\hline Region: Northeast & 0.282 & 0.152 & 1.856 & 0.063 & 0.363 & 0.248 & 1.466 & 0.143 \\
\hline Region: West & 0.776 & 0.196 & 3.959 & 0.000 & 0.974 & 0.255 & 3.817 & 0.000 \\
\hline \multicolumn{9}{|l|}{$W$} \\
\hline Foreign born & -0.463 & 0.555 & -0.834 & 0.405 & & & & \\
\hline Mother foreign born & 0.910 & 0.392 & 2.323 & 0.020 & & & & \\
\hline Mothers educ.: 12 & 0.652 & 0.181 & 3.601 & 0.000 & & & & \\
\hline Mothers educ.: 13 & 0.469 & 0.456 & 1.029 & 0.304 & & & & \\
\hline Mothers educ.: 14 & 0.554 & 0.478 & 1.159 & 0.247 & & & & \\
\hline Mothers educ.: 15 & 0.470 & 0.515 & 0.912 & 0.362 & & & & \\
\hline Mothers educ.: 16 & -0.053 & 0.379 & -0.141 & 0.888 & & & & \\
\hline Mothers educ.: 17 & 0.226 & 0.901 & 0.251 & 0.802 & & & & \\
\hline Mothers educ.: 18 or more & -0.498 & 0.685 & -0.728 & 0.467 & & & & \\
\hline Mothers educ. missing & -0.155 & 0.372 & -0.416 & 0.678 & & & & \\
\hline Fathers educ.: 12 & 0.414 & 0.216 & 1.918 & 0.055 & & & & \\
\hline Fathers educ.: 13 & 1.701 & 0.490 & 3.472 & 0.001 & & & & \\
\hline Fathers educ.: 14 & 0.992 & 0.427 & 2.324 & 0.020 & & & & \\
\hline Fathers educ.: 15 & 1.372 & 0.531 & 2.584 & 0.010 & & & & \\
\hline Fathers educ.: 16 & 1.516 & 0.301 & 5.041 & 0.000 & & & & \\
\hline Fathers educ.: 17 & 0.519 & 0.522 & 0.995 & 0.320 & & & & \\
\hline Fathers educ.: 18 or more & 2.132 & 0.403 & 5.294 & 0.000 & & & & \\
\hline Fathers educ. missing & -1.042 & 0.226 & -4.617 & 0.000 & & & & \\
\hline Constant & -0.410 & 0.419 & -0.977 & 0.328 & -0.356 & 0.642 & -0.555 & 0.579 \\
\hline
\end{tabular}

NOTE: The variables in $X$ are measured in 1998 and those in $W$ in 1979. 


\section{A.3 Propensity Score Distributions}
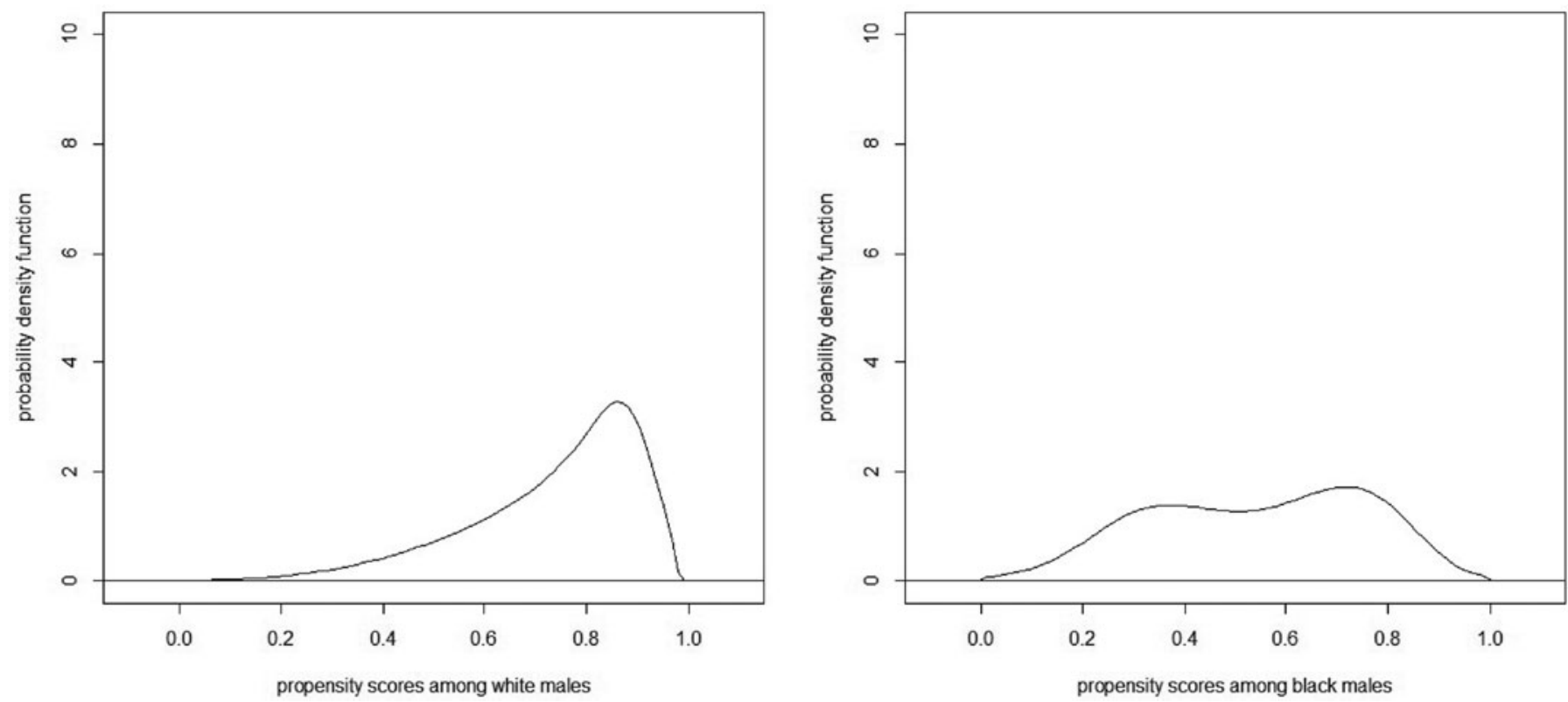

Figure 4. Density estimates of the estimates of $\operatorname{Pr}(G=1 \mid X)$ across groups. Note: density estimation is based on splines using the (default setting of the) logspline command for R. The lower and upper bounds of the support of the propensity scores are set to 0 and 1 .
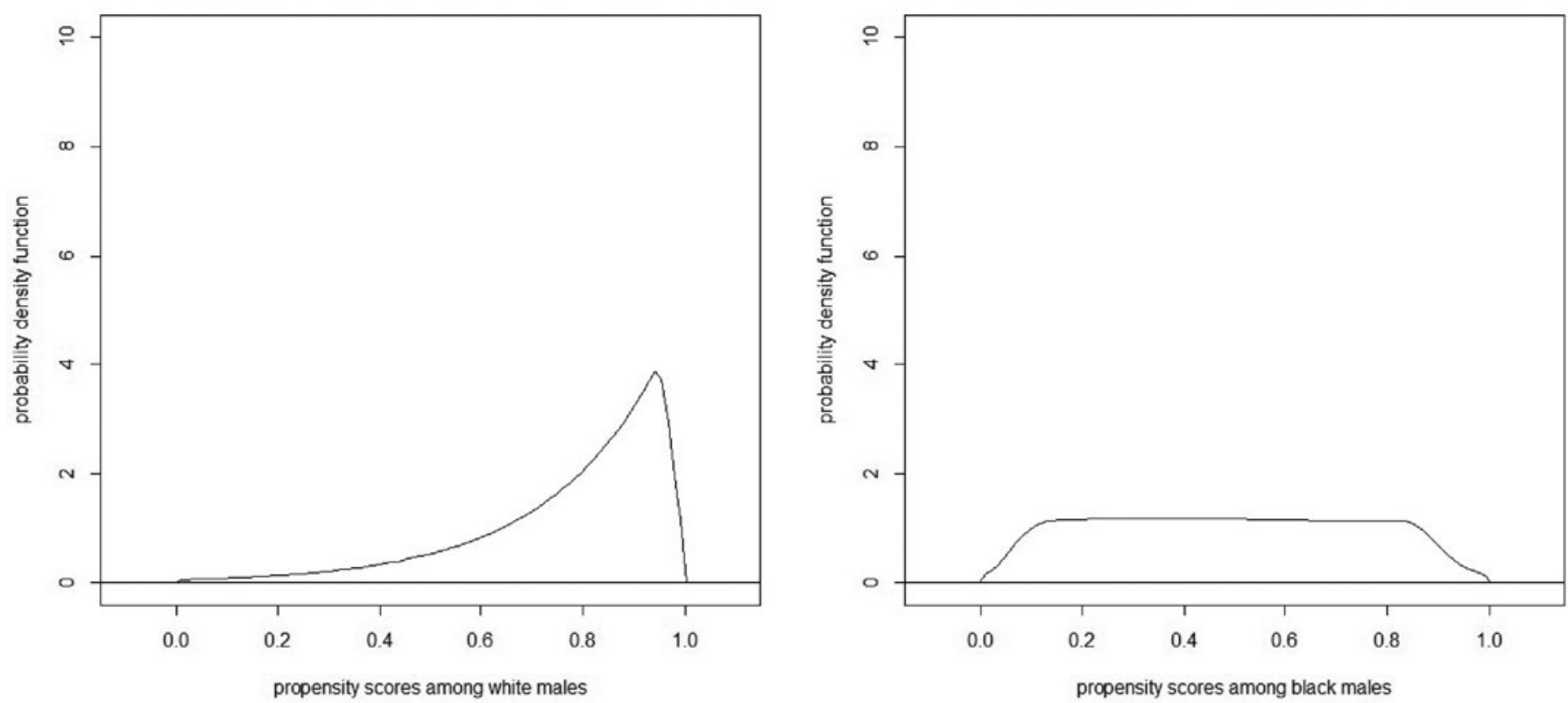

Figure 5. Density estimates of the estimates of $\operatorname{Pr}(G=1 \mid X, W)$ across groups. Note: density estimation is based on splines using the (default setting of the) logspline command for R. The lower and upper bounds of the support of the propensity scores are set to 0 and 1 . 


\section{ACKNOWLEDGMENTS}

I have benefited from comments by Christina Felfe, Michael Lechner, Andreas Steinmayr, and three anonymous referees.

[Received August 2013. Revised June 2014.]

\section{REFERENCES}

Albert, J. M., and Nelson, S. (2011), "Generalized Causal Mediation Analysis," Biometrics, 67, 1028-1038. [185,188]

Baron, R. M., and Kenny, D. A. (1986), "The Moderator-Mediator Variable Distinction in Social Psychological Research: Conceptual, Strategic, and Statistical Considerations," Journal of Personality and Social Psychology, 51, 1173-1182. [179]

Barsky, R., Bound, J., Charles, K., and Lupton, J. (2002), "Accounting for the Black-White Wealth Gap: A Nonparametric Approach," Journal of the American Statistical Association, 97, 663-673. [179,183]

Bayard, K., Hellerstein, J., Neumark, D., and Troske, K. (1999), "Why are Racial and Ethnic Wage Gaps Larger for Men Than for Women? Exploring the Role of Segregation Using the New Worker-Establishment Characteristic Database," National Bureau of Economic Research, Working Paper 6997. [186]

Bertrand, M., and Mullainathan, S. (2004), "Are Emily and Greg More Employable than Lakisha and Jamal? A Field Experiment on Labor Market Discrimination," The American Economic Review, 94, 991-1013. [180]

Blinder, A. (1973), "Wage Discrimination: Reduced Form and Structural Estimates," Journal of Human Resources, 8, 436-455. [179,181,185,187]

Cai, Z., Kuroki, M., Pearl, J., and Tian, J. (2008), "Bounds on Direct Effects in the Presence of Confounded Intermediate Variables," Biometrics, 64, 695-701. [185]

DiNardo, J., Fortin, N., and Lemieux, T. (1996), "Labor Market Institutions and the Distribution of Wages, 1973-1992: A Semiparametric Approach," Econometrica, 64, 1001-1044. [179]

Firpo, S., Fortin, N. M., and Lemieux, T. (2007), "Decomposing Wage Distributions Using Recentered Influence Functions Regressions," mimeo, University of British Columbia. [179]

_ (2009), "Unconditional Quantile Regressions," Econometrica, 77, 953973. [179]

Flores, C. A., and Flores-Lagunes, A. (2009), "Identification and Estimation of Causal Mechanisms and Net Effects of a Treatment under Unconfoundedness," IZA DP No. 4237. [183]

(2010), "Nonparametric Partial Identification of Causal Net and Mechanism Average Treatment Effects," mimeo, University of Florida. [185]

Frölich, M. (2007), "Propensity Score Matching Without Conditional Independence Assumption with an Application to the Gender Wage Gap in the United Kingdom," Econometrics Journal, 10, 359-407. [179]

Greiner, D. J., and Rubin, D. B. (2011), "Causal Effects of Perceived Immutable Characteristics," The Review of Economics and Statistics, 93, 775-785. [180]

Hirano, K., Imbens, G. W., and Ridder, G. (2003), "Efficient Estimation of Average Treatment Effects Using the Estimated Propensity Score," Econometrica, 71, 1161-1189. [184]

Huber, M. (2014), "Identifying Causal Mechanisms (Primarily) Based on Inverse Probability Weighting," Journal of Applied Econometrics, 29, 920 943. [180,184,188]

Imai, K., Keele, L., and Yamamoto, T. (2010), "Identification, Inference and Sensitivity Analysis for Causal Mediation Effects," Statistical Science, 25, 51-71. [180,181,182,183,184]

Imai, K., and Ratkovic, M. (2014), "Covariate Balancing Propensity Score," Journal of the Royal Statistical Society, Series B, 76, 243-263. [185,187]

Imai, K., Tingley, D., and Yamamoto, T. (2012), "Experimental Designs for Identifying Causal Mechanisms," Journal of the Royal Statistical Society, Series A, 176, 5-51. [185]

Imai, K., and Yamamoto, T. (2013), "Identification and Sensitivity Analysis for Multiple Causal Mechanisms: Revisiting Evidence From Framing Experiments," Political Analysis, 21, 141-171. [185,188]
Imbens, G. W. (2004), "Nonparametric Estimation of Average Treatment Effects Under Exogeneity: A Review," The Review of Economics and Statistics, 86, 4-29. [180]

Judd, C. M., and Kenny, D. A. (1981), "Process Analysis: Estimating Mediation in Treatment Evaluations," Evaluation Review, 5, 602-619. [179]

Juhn, C., Murphy, K., and Pierce, B. (1993), "Wage Inequality and the Rise in Returns to Skill," Journal of Political Economy, 101, 410-442. [179]

Kang, J. D. Y., and Schafer, J. L. (2007), "Demystifying Double Robustness: A Comparison of Alternative Strategies for Estimating a Population Mean From Incomplete Data," Statistical Science, 22, 523-539. [184]

Khan, S., and Tamer, E. (2010), "Irregular Identification, Support Conditions, and Inverse Weight Estimation," Econometrica, 78, 2021-2042. [184]

Kunze, A. (2008), "Gender Wage Gap Studies: Consistency and Decomposition," Empirical Economics, 35, 63-76. [183]

Lam, D., and Schoeni, R. F. (1993), "Effects of Family Background on Earnings and Returns to Schooling: Evidence from Brazil," Journal of Political Economy, 101, 710-740. [180]

Machado, J., and Mata, J. (2005), "Counterfactual Decomposition of Changes in Wage Distributions Using Quantile Regression," Journal of Applied Econometrics, 20, 445-465. [179]

MaCurdy, T., Mroz, T. and Gritz, R. M. (1998), "An Evaluation of the National Longitudinal Survey on Youth," The Journal of Human Resources, 33, 345436. [188]

Melly, B. (2005), "Decomposition of Differences in Distribution Using Quantile Regression," Labour Economics, 12, 577-590. [179]

Mora, R. (2008), "A Nonparametric Decomposition of the Mexican American Average Wage Gap," Journal of Applied Econometrics, 23, 463-485. [179]

Newey, W. K. (1984), "A Method of Moments Interpretation of Sequential Estimators," Economics Letters, 14, 201-206. [185]

Nopo, H. (2008), "Matching as a Tool to Decompose Wage Gaps," Review of Economics and Statistics, 90, 290-299. [179]

Oaxaca, R. (1973): "Male-Female Wage Differences in Urban Labour Markets," International Economic Review, 14, 693-709. [179,181,185,187]

O’Neill, J. E., and O’Neill, D. M. (2005), "What Do Wage Differentials Tell Us about Labor Market Discrimination?", Working Paper No. 11240, National Bureau of Economic Research. [186]

Pearl, J. (2001), "Direct and Indirect Effects," in Proceedings of the Seventeenth Conference on Uncertainty in Artificial Intelligence, San Francisco: Morgan Kaufman, pp. 411-420. [183]

Robins, J. M. (2003), "Semantics of causal DAG Models and the Identification of Direct and Indirect Effects," in Highly Structured Stochastic Systems, eds. P. Green, N. Hjort, and S. Richardson, Oxford: Oxford University Press, pp. 70-81. [183]

Robins, J. M., and Greenland, S. (1992), "Identifiability and Exchangeability for Direct and Indirect Effects," Epidemiology, 3, 143-155. [180]

Robins, J. M., and Richardson, T. (2010), "Alternative Graphical Causal Models and the Identification of Direct Effects," in Causality and Psychopathology: Finding the Determinants of Disorders and Their Cures, eds. P. Shrout, K. Keyes, and K. Omstein, Oxford: Oxford University Press. [185,188]

Rosenbaum, P. (1984), "The Consequences of Adjustment for a Concomitant Variable That has been Affected by the Treatment," Journal of Royal Statistical Society, Series A, 147, 656-666. [180]

Rosenbaum, P., and Rubin, D. (1983), "The Central Role of the Propensity Score in Observational Studies for Causal Effects," Biometrika, 70, 41-55. [180]

Rothe, C., and Firpo, S. (2013), "Semiparametric Estimation and Inference Using Doubly Robust Moment Conditions,” Discussion Paper No. 7564, IZA. [184]

Rubin, D. B. (1974), "Estimating Causal Effects of Treatments in Randomized and Nonrandomized Studies," Journal of Educational Psychology, 66, 688701. $[181,184]$

Waernbaum, I. (2012), "Model Misspecification and Robustness in Causal Inference: Comparing Matching With Doubly Robust Estimation," Statistics in Medicine, 31, 1572-1581. [184]

Yamamoto, T. (2013), "Identification and Estimation of Causal Mediation Effects With Treatment Noncompliance," unpublished manuscript, MIT Department of Political Science. [185]

Zheng, W., and van der Laan, M. J. (2012), "Targeted Maximum Likelihood Estimation of Natural Direct Effects," The International Journal of Biostatistics, 8, 1-40. [184] 